\title{
Fatores associados à carga de trabalho de enfermagem em Unidade de Terapia Intensiva*
}

\author{
FACTORS ASSOCIATED WITH NURSING WORKLOAD IN ADULT INTENSIVE CARE UNITS
}

\author{
FACTORES ASOCIADOS A LA CARGA DE TRABAJO DE ENFERMERÍA \\ EN UNIDAD DE TERAPIA INTENSIVA
}

\author{
Leilane Andrade Gonçalves', Kátia Grillo Padilha"
}

\section{RESUMO}

Os objetivos desta pesquisa foram analisar a carga de trabalho de enfermagem e os fatores associados a ela, no primeiro dia de internação dos pacientes na Unidade de Terapia Intensiva (UTI). Trata-se de um estudo retrospectivo, de corte transversal e com abordagem quantitativa, realizado em abril de 2002 e outubro de 2004. Os dados foram extraídos de um banco de dados que reuniu informações de 5 UTIs de dois hospitais privados e a amostra foi constituída por 214 pacientes adultos que permaneceram no mínimo 24 horas na UTI. A média do escore total do Nursing Activities Score (NAS) foi de $69,9 \%$ e mediana de $68,0 \%$. Verificou-se, segundo a mediana, que $109(50,9 \%)$ indivíduos tiveram alta carga de trabalho de enfermagem e 105 $(49,1 \%)$ baixa carga. Observouse também que a gravidade, a idade do paciente e o tipo de tratamento não foram fatores associados à demanda de trabalho de enfermagem.

\section{DESCRITORES}

Carga de trabalho.

Recursos humanos de enfermagem no hospital. Unidades de Terapia Intensiva.

\section{ABSTRACT}

This study was aimed both at analyzing the nursing workload on the first day of admission of patients in Intensive Care Units (ICUs) and the factors associated with it. This is a qualitative, retrospective, cross-section study that was carried out in April of 2002 and October of 2004. The data were taken from a database that gathered information from 5 ICUs from two private hospitals and the sample was comprised of 214 adult patients that remained in the ICU for at least 24 hours. The total Nursing Activities Score (NAS) average was $69.9 \%$, and the median $68.0 \%$. According to the median, it was verified that 109 $(50.9 \%)$ individuals required heavy nursing attention and the remaining $105(49.1 \%)$ required less attention. The severity of the illness, the patient's age and the kind of treatment were not factors associated with nursing workload in the first 24 hours at the ICU.

\section{KEY WORDS}

Workload.

Nursing staff, hospital. Intensive Care Units.

\section{RESUMEN}

Los objetivos de esta pesquisa fueron analizar la carga de trabajo de enfermería y los factores asociados a ella, en el primer día de internación de los pacientes en la Unidad de Terapia Intensiva (UTI). Se trata de un estudio retrospectivo, de corte transversal y con abordaje cuantitativa, realizado en abril de 2002 y octubre de 2004. Los datos fueron extraídos de un banco de datos que reunió informaciones de 5 UTIs de dos hospitales particulares y la muestra fue constituida por 214 pacientes adultos que permanecieron en el mínimo de 24 horas en la UTI. La media de escore total del Nursing Activities Store (NAS) fue de $69,9 \%$ y mediana de $68,0 \%$. Se verificó, según la mediana, que 109 $(50,9 \%)$ individuos tuvieron alta carga de trabajo de enfermería y $105(49,1 \%)$ baja carga. Se observó también que la gravedad, la edad del paciente y el tipo de tratamiento no fueron factores asociados a la demanda de trabajo de enfermería.

\section{DESCRIPTORES}

Carga de trabajo.

Personal de enfermería en

hospital.

Unidades de Terapia Intensiva.
Extraído da dissertação "Fatores associados à carga de trabalho de enfermagem em Unidade de Terapia Intensiva de adultos no primeiro dia de internação", Escola de Enfermagem, Universidade de São Paulo, 2006.

I Mestre em Enfermagem pela Escola de Enfermagem, Universidade de São Paulo (EEUSP). Enfermeira da UTI de Adultos do Hospital Sírio Libanês, São Paulo, SP, Brasil. jagon@ig.com.br

II Enfermeira. Professora Doutora Associada ao Departamento de Enfermagem Médico-Cirúrgico da Escola de Enfermagem, Universidade de São Paulo (EEUSP), São Paulo, SP, Brasil. kgpadilh@usp.br 


\section{INTRODUÇÃO}

Caracterizar a carga de trabalho de enfermagem em Unidade de Terapia Intensiva (UTI) com vistas a obter um quantitativo de pessoal que assegure qualidade e adequada relação custo-benefício da assistência intensiva é busca antiga que acompanha o próprio desenvolvimento histórico dessas unidades ${ }^{(1-2)}$.

Nas UTI(s), reconhecidas pela elevada carga, (que representa um fator de alto custo), e pelo elevado índice de mortalidade, (medida que expressa produção/rendimento) ${ }^{(3)}$, durante décadas utilizou-se a razão mortalidade/morbidade como parâmetro de escolha para descrever resultado da eficiência do cuidado intensivo. Todavia, nos últimos anos, a inclusão da demanda de trabalho de enfermagem como um parâmetro para avaliação dos resultados, tem sido também considerada, devido ao seu impacto na qualidade da assistência intensiva ${ }^{(3)}$.

Dessa forma, a avaliação de tal demanda de trabalho, bem como dos fatores associados a ela, tem se mostrado indispensável como recurso de gestão dessas unidades, visto que uma equipe superdimensionada implica em alto custo. Por outro lado, sabe-se que uma equipe reduzida tende a determinar uma queda na eficiência da assistência, prolongando a internação, aumentando a mortalidade/ morbidade e gerando um maior custo no tratamento dos pacientes ${ }^{(4)}$.

Nesse contexto, foi o desenvolvimento do Therapeutic Intervention Scoring System (TISS), em 1974, que introduziu na prática clínica em UTI a medida da carga de trabalho de enfermagem ${ }^{(5)}$.

Sendo um instrumento de medida pautado nas intervenções terapêuticas realizadas no doente em estado grave, a primeira versão do TISS foi revista e ampliada em 1983, passando a relacionar 76 intervenções terapêuticas ${ }^{(6)}$, ao invés das 57 inicialmente propostas. Decorridos 16 anos da existência do TISS-76, a nova simplificação dessa versão reduziu para 28 o número de intervenções contempladas, passando a ser denominado TISS-28 ${ }^{(7)}$.

Apesar da importância desses sistemas para uso específico em UTI, a aplicação prática mostrou falhas estruturais dos instrumentos para a medida total da demanda de trabalho, uma vez que as atividades relacionadas ao cuidado indireto do paciente, como tarefas organizacionais; as pausas no trabalho e outras atividades não estavam incluídas nas suas composições. Com vistas a superar as lacunas apontadas, um novo ajuste do TISS-28 foi proposto em $2003^{(8)}$.
Essa nova versão, conhecida como NAS (Nursing Activities Score), resultou de uma mudança expressiva do TISS-28, sobretudo na categoria atividades básicas, que passou a abarcar itens não contemplados anteriormente. Nesse formato, o novo sistema apresentou sensibilidade de $80.8 \%$ para a medida das atividades de enfermagem, superando a abrangência de 43,3\% do TISS-28, mostrando, desta forma, que o NAS descreve aproximadamente duas vezes mais o tempo gasto pela enfermagem no cuidado ao paciente em estado crítico quando comparado com o TISS- $28^{(8)}$.

Com essa nova estrutura, a pontuação obtida com o NAS expressa diretamente a porcentagem de tempo gasto pela equipe de enfermagem na assistência ao doente em estado crítico, em 24 horas, podendo chegar no máximo a $176,8 \%{ }^{(8)}$.

Na literatura são poucos os estudos que utilizaram o NAS. Talvez isso se justifique pelo fato de ser um instrumento novo, validado e adaptado recentemente para a realidade brasileira ${ }^{(9)}$. No entanto, observa-se um número crescente de enfermeiros, intensivistas ou gerenciais, que vêm aplicando o instrumento e divulgando os resultados em eventos científicos da área de terapia intensiva ${ }^{(9-10)}$.

A despeito da relevância das investigações voltadas à identificação das demandas de trabalho de enfermagem em UTI, pouco se conhece também a respeito dos fatores associados a essa carga de trabalho, quando mensuradas pelo NAS. Dentre eles, pode-se considerar de interesse os demográficos e clínicos como idade, sexo, dados da internação, como procedência, tipo de tratamento, tempo de permanência e condição de saída da UTI, além da gravidade.

O conhecimento dos fatores associados à elevada carga de trabalho de enfermagem é um recurso fundamental para o enfermeiro em sua prática na UTI. Pesquisas vêm sendo realizadas com o objetivo de identificar tais fatores durante a internação na UTI, porém, foram encontrados poucos estudos que fizessem esse tipo de análise no primeiro dia de internação.

Nessas circunstâncias, uma equipe de enfermagem com o conhecimento prévio dos fatores associados à alta carga de trabalho é capaz de estabelecer estratégias para admitir o paciente na Unidade e dar continuidade ao processo de cuidar no seu primeiro dia na UTI, de modo a garantir a qualidade e eficiência do seu trabalho. Dessa forma, considerandose a escassez de estudos sobre a demanda de trabalho de enfermagem mensurada pelo NAS e a necessidade de aprofundar o conhecimento sobre os fatores associados a essa demanda no primeiro dia de internação, optou-se pela realização deste estudo. 


\section{OBJETIVOS}

Geral: Analisar a carga de trabalho de enfermagem e identificar os fatores associados a ela no primeiro dia de internação dos pacientes na UTI.

Específicos: Caracterizar a carga de trabalho de enfermagem no primeiro dia de internação dos pacientes na UTI, segundo o NAS; descrever as intervenções terapêuticas realizadas nos pacientes, segundo o NAS; comparar a carga de trabalho de enfermagem entre os pacientes com diferentes características demográficas e clinicas, nas primeiras 24 horas de internação na UTI e verificar os fatores associados à alta carga de trabalho de enfermagem no primeiro dia de internação na UTI.

\section{MÉTODO}

Trata-se de um estudo de abordagem quantitativa, de corte transversal e retrospectivo. Os dados analisados nesta investigação foram extraídos de um banco de dados que reuniu informações de 5 UTI(s) de dois hospitais privados, de nível terciário, de grande e médio porte, do município de São Paulo, zona central da cidade. Das 5 UTI(s) estudadas, 4 eram do hospital de grande porte, sendo 2 UTI(s) gerais e 2 especializadas em neurologia, com 36 e 20 leitos, respectivamente e 1 UTI era do hospital de médio porte, geral, com 25 leitos.

A amostra, escolhida por conveniência, foi constituída por todos os pacientes admitidos nas UTI(s) no período da coleta de dados, com idade igual ou maior que 18 anos, submetidos a tratamento clínico ou cirúrgico e que nelas permaneceram internados por um período mínimo de 24 horas. Compuseram a amostra um total de 214 pacientes.

O projeto de pesquisa foi analisado e aprovado pelo Comitê de Ética em Pesquisa da Escola de Enfermagem da USP (Processo CEP n. 498/2005).

A coleta de dados nos dois hospitais foi realizada por enfermeiros intensivistas, treinados e orientados por uma docente da Escola de Enfermagem da Universidade de São Paulo (EEUSP), membro do Departamento Médico-Cirúrgico, com experiência na aplicação dos índices utilizados. Os dados foram coletados em abril de 2002 e outubro de 2004, nos hospitais de grande e médio porte, respectivamente, segundo o mesmo procedimento metodológico, por meio de três instrumentos padronizados.

A ficha de levantamento de dados utilizada para o registro dos dados demográficos e clínicos, do primeiro e último dia do paciente na UTI, foi constituída da seguinte forma: número de registro hospitalar do paciente; dados demográficos: idade e sexo; e dados clínicos: data da internação e saída da UTI, assim como o horário, motivo da internação, procedência (centro cirúrgico, pronto socorro, outro hospital ou outra UTI e demais unidades intra-hospitalares), tipo de tratamento (clínico ou cirúrgico) e condição de saída (sobrevivente ou não sobrevivente).

Os dados descritos nesse instrumento, em sua maioria, foram encontrados no prontuário do paciente. Todavia, na ausência de algumas informações, as mesmas foram fornecidas pelos enfermeiros da Unidade.

O índice NAS foi utilizado visando avaliar a carga de trabalho de enfermagem. A coleta do NAS foi realizada para cada paciente, utilizando-se a ficha de controle diário dos pacientes e, quando necessário, as informações relatadas pelos enfermeiros. $\mathrm{O}$ preenchimento do índice baseou-se nos dados referentes às primeiras 24 horas de internação na UTI, que se iniciaram na hora da chegada do paciente na Unidade.

O Simplified Acute Score (SAPS II) foi aplicado para avaliar a gravidade dos pacientes admitidos na UTI. A coleta do índice foi realizada tendo como base os dados referentes às primeiras 24 horas de internação na UTI. O instrumento foi aplicado para cada paciente e a maioria das informações estava contida no prontuário. Ressalta-se que os enfermeiros da Unidade participaram com o fornecimento dos dados apenas quando os mesmos não tinham sido registrados.

Os dados foram armazenados e analisados utilizando o programa SPSS (Statistical Package for the Social Sciences) versão 12.0 (SPSS, 2002), estabelecendo-se as análises descritivas e inferenciais pertinentes ao estudo, as quais foram realizadas por profissional capacitado.

Os indivíduos foram divididos em dois grupos, segundo a carga de trabalho de enfermagem. Para isso, foi considerada a mediana do NAS que foi de $68 \%$, separando os doentes em baixa carga $(\leq 68 \%)$ e alta carga de trabalho $(>68 \%)$.

Os pacientes também foram classificados segundo a gravidade, através da mediana do SAPS II, que foi de 43 pontos. Assim, os indivíduos com valores inferiores a 43 foram considerados de baixa gravidade e aqueles com escore maior ou igual a 43, de alta gravidade.

Estatísticas descritivas foram feitas para todas as variáveis estudadas. O teste de Kolmogorov-Smirnov foi utilizado para testar a hipótese de normalidade na distribuição das variáveis contínuas, considerando-se a distribuição normal quando valores de $\mathrm{p}$-valor foram maiores que $0,05^{(11)}$.

Os resultados foram considerados estatisticamente significantes se p-valor $<0,05$, com intervalo de $95 \%$ de confiança.

Para avaliar a associação entre a carga de trabalho de enfermagem (alta e baixa) e as variáveis demográficas e clínicas foi utilizado o teste de associação de Qui-quadrado de Pearson $\left(\chi^{2}\right)$. 
A análise de regressão logística foi utilizada visando identificar os fatores associados à alta carga de trabalho de enfermagem.

\section{RESULTADOS}

Os dados mostram que a maioria dos pacientes era do sexo masculino $(54,7 \%)$ e idosa $(53,7 \%)$, com idade igual ou maior que 60 anos, seguidos daqueles entre 31 e 59 anos $(38,3 \%)$. Apenas $8 \%$ dos pacientes tinham idade entre 18 e 30 anos. Verificou-se nesse estudo idade média de 59,7 $( \pm 18,6)$, mediana de 62 e variação de 18 a 99 anos.

Grande parte dos pacientes era procedente do centro cirúrgico (46,3\%). Em 32,2\% das admissões, os pacientes vieram das unidades de internação e semi-intensiva, em 14,5\% do pronto socorro e em $7 \%$ de outro hospital ou outra UTI.

Como tipo de internação, a maioria dos pacientes $(53,7 \%)$ foi submetida a tratamento clínico. Em 30,4\% dos pacientes as afecções neurológicas foram predominantes como motivo de internação na UTI, seguidos pelos distúrbios respiratórios e gastrointestinais $(19,6 \%$ cada) e cardiológicos $(15 \%)$. As afecções músculo-esqueléticas, renais e outros ocorreram em menor freqüência $(5,6 \%, 5,1 \%$ e 4,7\%, respectivamente).

Quanto à gravidade, constatou-se que 50,5\% dos doentes foram considerados de baixa gravidade e $49,5 \%$ de alta gravidade, quando utilizada a mediana para avaliação dessa variável. A média de pontuação do SAPS II foi de 44,9 (+17,5), enquanto que para o Risco de Mortalidade (RM), observouse média de $37,8( \pm 27,8)$, mediana de $31 \%$ e variação de 1 a $100 \%$. Em relação ao tempo de permanência na UTI, observou-se que a maioria $(73,4 \%)$ dos pacientes esteve na Unidade por período igual ou menor do que 6 dias. Os pacientes permaneceram, em média, $5,7( \pm 6,6)$ dias na UTI e mediana de 3 dias.

Em relação à condição de saída da Unidade, verificou-se que a maioria dos pacientes sobreviveu $(84,1 \%)$, enquanto que 34 (15,9\%) evoluíram a óbito.

Com relação à carga de trabalho de enfermagem, a média do escore total do NAS foi de $69,9 \%( \pm, 11,2)$, mediana de $68 \%$, variação de $54,3 \%$ a $122,5 \%$. As intervenções terapêuticas prevalentes, segundo o NAS, encontram-se descritas a seguir.

Em $100 \%$ dos pacientes foram realizadas as seguintes intervenções terapêuticas: investigações laboratoriais e medicação, exceto drogas vasoativas. Com freqüências menores, porém ainda elevadas, foram verificadas as intervenções: suporte e cuidados aos familiares e pacientes com dedicação exclusiva por cerca de 1 hora $(98,6 \%)$; medida quantitativa do débito urinário (ex. sonda vesical de demora) com $97,7 \%$; qualquer forma de ventilação mecânica ou assistida (96,3\%); realização de procedimentos de higiene de rotina $(88,3 \%)$; realização de tarefas administrativas e gerenciais como solicitação de exames, processamento de dados clínicos e troca de informações profissionais, que exigiram menos de duas horas, assim como monitorização e controles por 4 horas ou mais $(84,6 \%$, cada); tratamento para melhora da função pulmonar $(81,8 \%)$ e cuidados com drenos $(57,9 \%)$.

Quando comparadas às médias da carga de trabalho de enfermagem entre os pacientes com diferentes características sócio-demográficas observou-se que aqueles que foram a óbito $(\mathrm{p}=0,02)$ e os que permaneceram mais de seis dias na UTI $(p=0,00)$ apresentaram significativamente maior carga de trabalho de enfermagem nas primeiras 24 horas de internação na Unidade.

Após essas análises, investigou-se a correlação entre a carga de trabalho de enfermagem e as variáveis contínuas, encontrando correlação significativa apenas entre a carga de trabalho de enfermagem e o tempo de permanência dos indivíduos na UTI, tendo sido esta uma correlação fraca $(0,3)$.

A distribuição dos pacientes segundo a demanda de trabalho no primeiro dia de internação na UTI, considerandose a mediana do NAS (68\%), encontra-se na Figura 1.

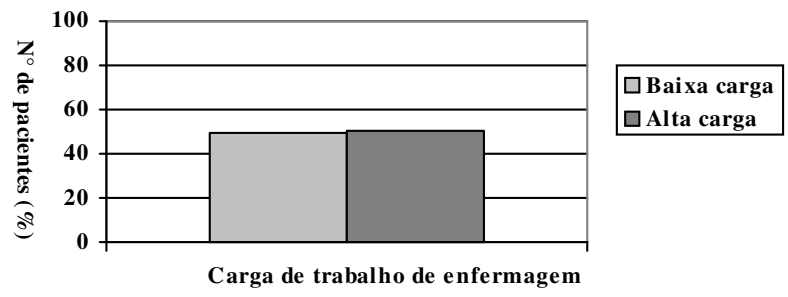

Figura 1 - Distribuição do percentual de pacientes internados na UTI no primeiro dia de internação segundo alta e baixa carga de trabalho de enfermagem - São Paulo - 2002-2004

Conforme mostra a Figura 1, do total da amostra (214) verificou-se que, embora a maioria dos pacientes, ou seja, $109(50,9 \%)$ tivesse exigido alta carga de trabalho de enfermagem no primeiro dia de internação na UTI, foi muito próximo o número de doentes (105-49,1\%), que demandaram baixa carga.

Ao serem analisados os grupos de pacientes que apresentaram alta e baixa carga de trabalho de enfermagem com as variáveis estudadas, observou-se apenas associação com o tempo de permanência $\left(\mathrm{X}^{2}=7,7, \mathrm{p}\right.$ valor $\left.=0,01\right)$, ou seja, dentre os pacientes que permaneceram mais de 6 dias na UTI, a maioria $(66,7 \%)$ demandou elevada carga no primeiro dia de internação.

Quando realizado análise de regressão univariada, verificou-se que pacientes que permaneceram na UTI por tempo igual ou superior a seis dias tiveram quase duas vezes e 
meia mais chance de exigir maior tempo de assistência de enfermagem no primeiro dia de internação do que os indivíduos com menor tempo de permanência.

Ao ser realizada a análise de regressão logística múltipla, verificou-se que mesmo na presença da condição de saída, o tempo de permanência manteve-se como fator de risco para alta carga de trabalho. Assim, pode-se concluir que o tempo de permanência na UTI foi um fator significativamente associado à elevada carga de trabalho de enfermagem no primeiro dia de internação.

\section{DISCUSSÃO}

Os resultados referentes às características demográficas investigadas neste estudo assemelham-se a maioria das pesquisas realizadas em UTI. Da amostra estudada (214), a maioria $(54,7 \%)$ pertencia ao sexo masculino. Estudos brasileiros, ao longo do tempo, vêm confirmando tal achado, com resultados que variaram de $54 \%$ a $58 \%{ }^{(9,12)}$.

Ao se analisar a idade da população estudada, verificou-se que a maioria $(53,7 \%)$ era composta por idosos. Pesquisas realizadas no Brasil confirmam esses achados. Em 1990, observou-se que $50,5 \%$ da população de duas UTI(s) pertencia à faixa etária entre 61 e 80 $\operatorname{anos}^{(13)}$ e em 2004, indivíduos com idade acima de 60 anos totalizaram $53 \%{ }^{(11)}$. Destaca-se que estudos internacionais também reiteram a predominância de idosos em suas amostras ${ }^{(14-15)}$.

A população está envelhecendo e com isso o número de pessoas com doenças crônico-degenerativas vêm aumentando ${ }^{(16)}$, desencadeando agravos à saúde com conseqüente necessidade de intervenções que requerem internação em UTI.

No que se refere aos dados clínicos, foi verificado que grande parte dos pacientes $(46,3 \%)$ era procedente do centro cirúrgico. Em 1990, no Brasil, constatou-se que dos pacientes internados em duas UTI(s) do Município de São Paulo, 34,9\% eram provenientes do centro cirúrgico, na instituição particular e $50 \%$ do pronto socorro, na instituição públi$\mathrm{ca}^{(13)}$ e em 1996, em 10 UTI(s) de três estados brasileiros foram encontrados $35,7 \%$ das admissões advindas do centro cirúrgico ${ }^{(17)}$.

Quanto ao tipo de tratamento, a maioria dos pacientes $(53,7 \%)$ foi internada na UTI por razões clínicas. Em 2002, um estudo encontrou admissões por motivos clínicos em $57 \%$ dos indivíduos ${ }^{(9)}$; já em 2004 e 2005, no Brasil, estudos também confirmaram tal achado em $78 \%$ e $60 \%$ das admissões, respectivamente ${ }^{(12,18)}$.

Como motivos de internação mais freqüentes encontraram-se neste estudo problemas neurológicos $(30,4 \%)$, se- guidos de respiratórios, gastrointestinais $(19,6 \%$, cada) e cardiológicos $(15 \%)$, igualmente ao verificado em outras investigações $^{(13,19)}$, incluindo pesquisa realizada pelo segundo Censo da Associação de Medicina Intensiva Brasilei$\mathrm{ra}^{(20)}$, que constatou como causas de admissão prevalentes as doenças dos sistemas cardiovascular e respiratório.

No que se refere à condição de saída da UTI, observouse uma mortalidade de $15,9 \%$, compatível com estudos estrangeiros que oscilaram entre 8 e $19 \%{ }^{(8,21)}$. Porém, diferente desses resultados, estudo brasileiro desenvolvido em um hospital universitário, no ano de 2002, verificou altas taxas de mortalidade, em torno de $33 \%{ }^{(12)}$, dados referendados por outros estudos nacionais que obtiveram taxas entre $29,0 \%$ e $35,0 \%{ }^{(22-23)}$.

Frente à mortalidade encontrada, abaixo da verificada em diversos estudos nacionais, há que se considerar que as próprias características da clientela atendida pelos hospitais, campos deste estudo, contribuem para uma melhor resposta ao tratamento e, conseqüentemente, menor mortalidade, apesar de a maioria dos pacientes ser idosa.

Quanto à gravidade dos pacientes nas primeiras 24 horas de internação na UTI, encontrou-se uma média SAPS II de 44,9 pontos, mediana de 43, variação entre 9 e 119 pontos e risco de mortalidade de $37,8 \%$.

A gravidade neste estudo, quando comparada às demais pesquisas nacionais e internacionais, é considerada elevada. A Média SAPS II, próxima a encontrada nesta pesquisa, foi constatada em estudo realizado em um hospital de características semelhantes ao desta investigação, com 41,7 pontos $^{(18)}$. Outros estudos brasileiros, no entan-to, encontraram média de SAPS II entre 27 e 33 $\operatorname{pontos}^{(24-25)}$.

Apesar de grande parte dos pacientes ser procedente do centro cirúrgico, a alta porcentagem de idosos internados para tratamento clínico e vindos das unidades de internação e semi-intensiva podem justificar a elevada gravidade encontrada nesta investigação.

Em relação ao tempo de permanência na UTI, a maioria dos pacientes $(73,4 \%)$ permaneceu por menos de 6 dias, com uma média de 5,7 dias. Esse resultado coaduna-se com o verificado no segundo Censo Brasileiro de UTI(s), realizado pela Associação de Medicina Intensiva Brasileira $(\mathrm{AMIB})^{(20)}$, em que o tempo médio de permanência variou de 3 a 6 dias, com predomínio de uma média entre 3 e 4 dias, assim como com os achados de diversos estudos nacionais e internacionais $^{(22,26)}$.

Referente à caracterização da carga de trabalho de enfermagem no primeiro dia de internação na UTI, observou-se neste 
estudo uma média elevada do escore total do NAS de cerca de $70 \%$ (69,9\%), com mínimo de 54,3\% e máximo de $122,5 \%$.

Considerando que o tempo gasto pela equipe de enfermagem para o cuidado de um paciente, no primeiro dia de internação na UTI, é cerca de $70 \%$, esses resultados trazem subsídios consistentes para demonstrar a elevada carga de trabalho nesse período. Tais informações são também fundamentais para o dimensionamento de pessoal e alocação de recursos humanos, sobretudo quando se busca maior segurança e satisfação do paciente como resultado da assistência intensiva. De acordo com os resultados encontrados nas UTI(s) estudadas, a relação técnico de enfermagem/paciente de 1:2, preconizada pela Portaria do Ministério da Saúde $n^{\circ} 3432$ de 12 de agosto de $1998^{(27)}$, seria insuficiente para o cuidado dos pacientes.

A soma das atividades terapêuticas discutidas neste estudo, segundo o NAS, representa a carga de trabalho de enfermagem que, por sua vez, foi analisada segundo as variáveis demográficas e clínicas, no primeiro dia de internação na UTI. Essa análise foi realizada considerando tanto a média como a mediana do índice, sendo essa última utilizada para classificar como alta e baixa a carga de trabalho de enfermagem.

Comparando-se a média da carga de trabalho com tais variáveis, observou-se que apenas a condição de saída da UTI $(p=0,02)$ e o tempo de permanência na Unidade $(\mathrm{p}=0,00)$ apresentaram diferenças estatisticamente significativas. Pacientes que evoluíram a óbito e que permaneceram mais de seis dias na UTI exigiram maior tempo de assistência de enfermagem, quando comparados com os sobreviventes e com pacientes que permaneceram menos tempo na Unidade.

Já quando utilizada a mediana do NAS para comparar os grupos correspondentes à alta e baixa carga com as variáveis estudadas, o tempo de permanência foi o único que apresentou associação significativa com a carga de trabalho $(\mathrm{p}=0,01)$. Tal resultado mostrou que dentre os pacientes que permaneceram mais de seis dias na UTI, a maioria $(66,7 \%)$ apresentou alta carga de trabalho de enfermagem nas primeiras 24 horas de internação.

Os doentes com maior tempo de permanência na UTI e que evoluem a óbito, geralmente, quando admitidos na Unidade, são considerados graves e instáveis, necessitando, na maioria das vezes, de terapêuticas complexas ${ }^{(12,24)}$. Por outro lado, neste estudo, observou-se que a gravidade dos pacientes não interferiu significativamente na carga de trabalho de enfermagem, quando utilizada a média e a comparação dos grupos, segundo a mediana do NAS.

Da mesma forma que a gravidade, o sexo, a idade, a procedência, o tipo de tratamento e os motivos de internação não apresentaram associação significativa com a demanda de trabalho de enfermagem.
Se a gravidade dos pacientes não apresentou associação significativa com a carga de trabalho de enfermagem, da mesma forma que as demais variáveis demográficas e clínicas levantadas como hipótese, quais são os fatores que realmente interferem na alta carga de trabalho, além do tempo de permanência e condição de saída?

Ao se investigar estes fatores entre as variáveis, observou-se que o único fator associado foi o tempo de permanência do paciente na UTI, mesmo quando ajustado para a condição de saída da Unidade. Os pacientes que permaneceram mais de 6 dias apresentaram quase duas vezes e meia mais risco de demandar alta carga de trabalho de enfermagem no primeiro dia de internação do que os indivíduos com menor tempo.

A necessidade de planejamento da assistência de enfermagem que será prestada ao doente em estado crítico no primeiro dia de internação na UTI impulsionou a investigação dos fatores preditivos da alta carga de trabalho que, por sua vez, não foi suficiente para a realização de tal previsão.

A relação entre dependência do paciente e carga de trabalho de enfermagem vem sendo cada vez mais investigada. Mudanças no grau de dependência interferem na carga de trabalho ${ }^{(28)}$. A dependência do paciente, avaliada, por exemplo, pelo nível de confusão e agitação psicomotora do doente em estado crítico, interfere na demanda de trabalho, tendo em vista que, uma vez presentes, o tempo de cuidado estabelecido para as atividades de enfermagem aumenta ${ }^{(29)}$.

Indivíduos em estado grave, inconscientes, imóveis e com todas as suas necessidades fisiológicas monitorizadas por suporte tecnológico, quando admitidos na UTI, podem requerer menos tempo de assistência de enfermagem quando comparados com pacientes ansiosos, assustados, estressados com o ambiente e capazes de comunicar dor e desconforto ${ }^{(30)}$.

A ausência de associação entre a carga de trabalho de enfermagem e as variáveis gravidade, idade e tipo de tratamento, no primeiro dia de internação na UTI, indica a necessidade de se explorar tal dependência como possível preditora da alta carga de trabalho de enfermagem. Assim, sistemas de classificação de dependência como estratégia para categorizar os doentes de acordo com a complexidade de cuidados que eles exigem da equipe de enfermagem devem ser utilizados ${ }^{(30)}$.

Classificar os pacientes de acordo com estes sistemas tem sido considerada por diversos autores ${ }^{(31-32)}$ uma medida confiável para se estimar a demanda de trabalho de enfermagem, uma vez que as condições do paciente e, conseqüentemente as suas necessidades de cuidado são levadas em consideração.

O modelo de dependência do paciente permite com acurácia analisar as atividades de enfermagem e predizer 
carga de trabalho de enfermagem ${ }^{(33)}$. Outros autores ${ }^{(34)}$ também acreditam na avaliação da dependência do doente crítico como uma das melhores formas de se medir a carga de trabalho e, consequientemente de prevê-la.

Em síntese, os resultados desta investigação trazem contribuição importante. Permitem, sobretudo, contrapor-se a afirmações frequientes dos enfermeiros de UTI que solicitam maior quantidade de pessoal de enfermagem associando-a a gravidade dos pacientes em estado crítico.

No entanto, outros estudos precisam ser realizados com o objetivo de explorar este tema, tendo em vista as limitações desta pesquisa. Destacam-se dentre elas, o tipo de amostra, ou seja, de escolha por conveniência, realizada em apenas duas instituições, assim como o tamanho da amostra, que impedem a generalização dos resultados. Além disso, o número reduzido de estudos com a utilização do NAS ainda traz questionamentos sobre a sua adequação para medir a carga de trabalho de enfermagem em UTI. Adiciona-se a isso, a importância da utilização de outros instrumentos de medida de gravidade e de morbidade em estudos futuros como contribuição aos já existentes conhecimentos relacionados ao tema.

\section{CONCLUSÕES}

A carga de trabalho de enfermagem exigida pelos pacientes, que foram predominantemente do sexo masculino $(54,7 \%)$, idosos $(53,7 \%)$, submetidos a tratamento clínico $(53,7 \%)$, procedentes do centro cirúrgico $(46,3 \%)$, com baixa gravidade $(50,5 \%)$ e baixa taxa de mortalidade $(15,9 \%)$, foi elevada, com média do escore total do NAS de cerca de $70 \%$.

\section{REFERÊNCIAS}

1. Bloom BS, Lundberg D. Intensive care: where are we? Int J Technol Assess Health Care. 1992;8(3):379-81.

2. Dragsteed L, Qvist J. Epidemiology of intensive care. Int J Technol Assess Health Care. 1992;8(3):395-407.

3. Jakob SM, Rothen HU. Intensive care 1980-1995: change in patient characteristics, nursing workload and outcome. Intensive Care Med. 1997;23(11):1165-70.

4. Gaidzinski RR. Dimensionamento de pessoal de enfermagem em instituições hospitalares [tese livre-docência]. São Paulo: Escola de Enfermagem, Universidade de São Paulo; 1998.

5. Cullen DJ, Civetta JM, Briggs BA, Ferraro LC. Therapeutic Intervention Scoring System: a method for quantitative comparison of patient nurse. Crit Care Med. 1974;2(2):57-60.

6. Keene AR, Cullen DJ. Therapeutic intervention scoring system: update 1983. Crit Care Med. 1983;11(1):1-3.
No primeiro dia de permanência na UTI a maioria dos pacientes necessitou das seguintes atividades terapêuticas de enfermagem: investigações laboratoriais (100\%) e uso de medicações (100\%), suporte e cuidados aos familiares e pacientes, com dedicação exclusiva por cerca de 1 hora $(98,6 \%)$, medida quantitativa do débito urinário $(97,7 \%)$, qualquer forma de ventilação mecânica ou assistida $(96,3 \%)$, realização de procedimentos de higiene de rotina $(88,3 \%)$, de tarefas administrativas e gerenciais com duração inferior a duas horas e monitorização e controles por 4 horas ou mais em algum plantão $(84,6 \%)$, tratamento para melhora da função pulmonar $(81,8 \%)$, além de cuidados com drenos $(57,9 \%)$.

A comparação da carga de trabalho de enfermagem, segundo a média encontrada entre pacientes com diferentes características demográficas e clínicas, mostrou que aqueles que permaneceram na UTI por mais de 6 dias e que evoluíram a óbito foram os únicos que exigiram significativamente maior tempo da assistência de enfermagem no primeiro dia de internação na Unidade. Já quando analisados os grupos de pacientes que apresentaram alta e baixa carga de trabalho com as variáveis estudadas, observou-se associação significativa apenas com o tempo de permanência, ou seja, dentre os pacientes que permaneceram mais de 6 dias na UTI, a maioria demandou elevada carga no primeiro dia de internação.

O único fator preditor da elevada carga de trabalho de enfermagem na UTI foi o tempo de permanência na Unidade. Características como idade, tipo de tratamento, gravidade e demais variáveis não interferiram na demanda de trabalho de enfermagem no primeiro dia de internação do paciente na UTI.

7. Miranda DR, Rijk AD, Schaufeli W. Simplified therapeutic intervention scoring system: the TISS-28 itens-results from a multicenter study. Crit Care Med. 1996;24(1):64-73.

8. Miranda DR, Raoul N, Rijk A, Schaufeli W, Iapichino G. Nursing activities score. Crit Care Med. 2003;31(2):374-82.

9. Queijo AMG. Tradução para o português e validação de um instrumento de medida de carga de trabalho de enfermagem em unidade de terapia intensiva: Nursing Activities Score (NAS) [dissertação]. São Paulo: Escola de Enfermagem, Universidade de São Paulo; 2002.

10. Gonçalves LA, Garcia PC, Tofolleto MC, Padilha KG, Telles SCR. Necessidades de cuidados de enfermagem em Unidade de Terapia Intensiva: evolução diária dos pacientes segundo o (NAS) Nursing Activities Score. Rev Bras Enferm. 2006; 59(1):56-60.

11. Costa Neto PLO. Estatística. São Paulo: Edgard Blucher; 1977. 
12. Ducci AJ, Padilha KG. Gravidade de pacientes e demanda de trabalho de enfermagem em Unidade de Terapia Intensiva: análise evolutiva segundo o TISS-28. Rev Bras Ter Int. 2004; 16(1):22-7.

13. Pierin AMG, Padilha KG, Cruz DALM. Caracterização dos pacientes de duas unidades de terapia intensiva (UTI): condições sociais, processo de internação e intervenções terapêuticas. Rev Esc Enferm USP. 1990;24(3):371-88.

14. Castillo EL, Rivera FR, Rodriguez EM, Vazquez MG. TISS-76 and TISS-28: correlation of two therapeutic activity indices on a Spanish multicenter ICU database. Intensive Care Med. 2000;26(1):57-61.

15. Lefering R, Zart M, Neugebauner EAM. Retrospective evaluation of the Simplified Therapeutic Intervention Scoring System (TISS-28) in a surgical intensive care unit. Intensive Care Med. 2000;26(12):1794-802.

16. Instituto Brasileiro de Geografia e Estatística (IBGE). Censo demográfico 2000 [texto na Internet]. Rio de Janeiro; 2000 [citado 2006 maio 25]. Disponível em: http://www.ibge.gov.br/ home/estatistica/populacao/default_censo_2000.shtm.

17. Bastos PG, Sum X, Wagner DP, Knaus WA, Zimmerman JE. Application of the APACHE III prognostic system in Brazilian intensive care units: a prospective multicenter study. Intensive Care Med. 1996;22(6):564-70.

18. Conishi RMY. Avaliação do NAS - Nursing Activities Score como instrumento de medida da carga de trabalho de enfermagem em UTI geral adulto [dissertação]. São Paulo: Escola de Enfermagem, Universidade de São Paulo; 2005.

19. Silva MCM, Sousa RMC. Caracterização dos pacientes adultos e adolescentes das Unidades de Terapia Intensiva do município de São Paulo. Rev Paul Enferm. 2002;21(1):50-7.

20. Associação de Medicina Intensiva Brasileira (AMIB). $2^{\circ}$ Anuário Brasileiro de UTI's: 2002-2003. São Paulo; 2004.

21. Moreno R, Morais P. Validation of the simplified therapeutic intervention scoring system on na idependent database. Intensive Care Med. 1997;23(6):640-4.

22. Paiva SAR, Matai O, Resende NO, Campana AO. Análise de uma população de doentes atendidos em unidade de terapia intensiva: estudo observacional de sete anos (1992-1999). Rev Bras Ter Intensiva. 2002;14(2):73-80.
23. Telles SCR. Custo de pessoal na assistência direta de enfermagem em Unidade de Terapia Intensiva [dissertação]. São Paulo: Escola de Enfermagem, Universidade de São Paulo; 2003.

24. Nascimento EFA. Evolução da gravidade de pacientes adultos internados em uma Unidade de Terapia Intensiva. [dissertação]. São Paulo: Escola de Enfermagem, Universidade de São Paulo; 2002.

25. Livianu J, Orlando JMC, Proença JO. Comparação de 3 índices de gravidade de pacientes de Unidade de Terapia Intensiva. In: Anais do $7^{\circ}$ Congresso Brasileiro de Terapia Intensiva Adulto e Pediátrica; 1997; Salvador. São Paulo: AMIB; 1997. p. 141.

26. Miranda DR, Ryan DW, Scaufeli WB, Fidler V, editors. Organization and management of intensive care: a prospective study in european countries. Berlin: Spring; 1998. (Update in Intensive Care and Emergency Medicine, 29).

27. Brasil. Ministério da Saúde. Portaria n. 3432, de 12 de agosto de 1998. Estabelece critérios de classificação para as unidades de tratamento intensivo - UTI. Diário Oficial da União, Brasília, 13 ago. 1998. Seção 1, p.108-10.

28. Proctor S. Subjectivity and objectivity: the measurement of nursing workload. J Clin Nurs. 1991;1(1):123-9.

29. Carayon P, Gurses AP. A human factors engineering conceptual framework of nursing workload and patient safety in intensive care units. Intensive Crit Care Nurs. 2005;21(5):284-301.

30. Adomat R, Hewison A. Assessing patient category/dependence systems for determining the nurse/patient ratio in ICU and HDU: a review of approaches. J Nurs Manag. 2004;12(5): 299-308.

31. Campbell T, Taylor S, Callaghan S, Shuldham C. Case mix type as a predictor of nursing workload. J Nurs Manag. 1997; 5(4): 237-40.

32. Hurst K. Relationship between patient dependency, nursing workload and quality. Int J Nurs Stud. 2005;42(1):75-84.

33. James G. Nursing precious resources. Health Serv J. 1991; 101(5252):24-5.

34. Needham J. Accuracy and workload measurement: a fact or fallacy? J Nurs Manag. 1997;5(2):83-7. 